\title{
On the Economical Impacts of Cloud Computing in Information Technology Industry
}

\author{
Asst. Prof. Dr. Ediz Şaykol (Beykent University, Turkey)
}

\begin{abstract}
Cloud computing, as defined by United States National Institute of Standards and Technology (NIST), “... is a model for enabling convenient, on-demand network access to a shared pool of configurable computing resources (e.g., networks, servers, storage, applications, and services) that can be rapidly provisioned and released with minimal management effort or service provider interaction." Goal of the study is to highlight the positive economical impacts of cloud computing for companies, which can be assessed by focusing on their business goals to make more profit. Under the cloud computing model, firms can rent as many virtual machines as they need at any given time, and either design or use off-the-shelf solutions to integrate company-wide data and then easily distribute access to users within and outside of the company. Hence, cloud computing converts fixed capital costs to variable costs, prevents under or over provisioning, and allows minute by minute flexibility. Hence, the companies concentrate on their own business goals on top on a stable and effective information technology backbone. Most literature on cloud computing reside in computer science resources. However, there is little work focusing on the social and economic significance of cloud computing in the economics literature. Hence, this paper first discusses the basics of the cloud computing along with its implications on information technology (IT) field, with paying special attention to economical aspects. Then, empirical results and elaborations are to be given to yield a conclusion.
\end{abstract}

\section{Introduction}

Cloud Computing can be defined in various ways. The definition given by the National Institute of Standards and Technology has focused on hardware and management views. As stated, it is a model for enabling “... convenient, on-demand network access to a shared pool of configurable computing resources (e.g., networks, servers, storage, applications and services) that can be rapidly provisioned and released with minimal management effort or service provider interaction". Even though cloud computing is often considered as a technological term, it is actually a critical adoption in the business models for forecasting the use of the information technology (IT) to reduce cost in a general sense. As NIST implies, this cost savings can only be realized through the use of significant pooling of these "configurable computing resources" or resource pooling. According to NIST, resource pooling is the ability of a cloud to serve multiple customers with different physical and virtual resources which have been mapped dynamically (Bayrak, 2011).

In older times, the industry built their own server centers as many as needed to satisfy the demand. It is quite clear that it was time consuming since the hardware and software solutions co-exist in the server centers. Moreover, it is quite inflexible and requires more upfront investment to build up those server centers. However, cloud computing brings together existing technologies introducing service oriented architecture, distributed grid computing, virtualization and broadband networking to provide software, infrastructure, and platforms as services. That is the main fact that cloud computing has a deep impact on IT companies.

The companies in IT industry can now rent servers, instead of building its own centers under the cloud computing model. They can also use third party solutions to create a collaboration environment for their company-wide data, since mechanisms are generally provided to manage the access rights of the users/employers. Hence, cloud computing converts fixed capital costs to variable costs, and prevents under or over provisioning via providing flexibility. Moreover, from the supply perspective, the cloud computing paradigm provides economies of scale, distribution of costs among a large pool of users, centralization of infrastructures in areas with lower costs, and improved resource utilization contributes to value creation and efficiency improvements. Hence, cloud computing shares the same ideas of a disruptive general purpose technology to impact the economy as a whole with the efficiency improvements and large savings in operational costs as well as upfront capital costs of tech-startups (Armbrust et al., 2009).

In this paper, some preliminary information on cloud computing is provided in Section 2 for those who are unfamiliar to the basics of IT. Section 3 briefly discusses the economical impacts of cloud computing. Finally, Section 4 concludes the paper.

\section{Preliminaries on Cloud Computing}

Quite interestingly, even though cloud computing has been a trendy topic in the recent years (eg, (Hamilton, 2008) (Cheng, 2008) (Siegele, 2008)), there is still something unclear on its exact definition and its needs among the big players. The following three examples given in (Armbrust et al., 2009) worth revisiting here: 
- Larry Ellison, the CEO of Oracle, quoted in the Wall Street Journal, September 26, 2008, that "The interesting thing about Cloud Computing is that we've redefined Cloud Computing to include everything that we already do. . . . I don't understand what we would do differently in the light of Cloud Computing other than change the wording of some of our ads."

- Andy Isherwood, Hewlett-Packard's Vice President of European Software Sales, quoted similarly in ZDnet News, December 11, 2008, that "A lot of people are jumping on the [cloud] bandwagon, but I have not heard two people say the same thing about it. There are multiple definitions out there of "the cloud'."

- Richard Stallman thinks Cloud Computing is a trap for users - if applications and data are managed "in the cloud", users might become dependent on proprietary systems whose costs will escalate or whose terms of service might be changed unilaterally and adversely.

On the other hand, in an older paper (Parkhill, 1966), the 'computing' term is considered as a 'utility', such as electricity. However, being a utility requires loose coupling between the computing operation and the hardware corresponding to resources. As the cloud computing term has emerged, it is now possible that computing can be visualized as a utility.

\subsection{Cloud Computing Service Models}

Cloud Computing refers to both the applications delivered as services over the Internet and the hardware and systems software in the data centers that provide services to access, integrate, collaborate regarding the company-wide data. Providing services part of the software to Access data have long been referred to as 'Software as a Service (SaaS)'. Hence, the term 'cloud' is used to clarify the data is not actually there, it is 'inthe-air'. In short, the datacenters along with their hardware and software is a Cloud.

A cloud is classified with respect to the accessibility of the data served in the cloud. When a Cloud is made available to the public, we call it a Public Cloud; possibly the service is made available in a pay-as-you-go manner, and what is literally sold is Utility Computing. Current examples of public Utility Computing include Amazon Web Services, Google App Engine, and Microsoft Azure. A Private Cloud is used to refer to internal datacenters of a business or other organization that are not made available to the public (Armbrust et al., 2009).

The advantages of SaaS not only to end users but also to service providers are generally clear in minds. First of all, the service providers facilitated their software installation and maintenance processes, and hence centralized control over versioning. Similarly, the end users can access the service in an ubiquitous manner, in other words "anytime, anywhere". End users also easily share data and perform collaborations easily, since the data is kept safely in-the-air.

What Cloud Computing augment to SaaS is the fact that cloud computing gives alternatives to the providers so that they can deploy their product in the market without bothering the requirements of an appropriate data center. Companies deploy via SaaS even without actually building a data center. This actually means that the problems or inefficiencies of an owned data center are delegated (in fact transferred) to the cloud provided by the cloud user. The companies providing SaaS are the Cloud Users, and the companies who created a cloud infrastructure and selling usage are Cloud Providers.

From cloud providers' perspective, there are four major ways to adopt cloud computing technology:

- First one is a private cloud, in which the deployment the cloud infrastructure is operated for a single organization.

- Second, a community cloud is when the cloud infrastructure is shared by several organizations. It also supports a specific community that has shared interests. These cloud infrastructures may be managed by the organizations themselves or a third party.

- Third, a public cloud involves the cloud infrastructure being made available to the general public or a large industry group and is typically deployed by a separate organization selling cloud services.

- Finally, a hybrid cloud is a composition of two or more deployment models that are bound together by a technology that enables data and application portability.

The term Everything as a Service (XaaS) has been associated with many core services offered by cloud providers, including software (SaaS), development platforms (PaaS), computing infrastructure (IaaS), communication (CaaS), and data storage (DaaS), however, the most widely accepted classifications of service models focus on software, platform and infrastructure layers as the primary service models (Creeger (2009), Durkee (2010), Lin et al. (2009), Mell \& Grance (2009), Viega (2009), Vaguero et al. (2009), Weinhardt et al.(2009)).

There are three widely accepted primary service models where different capabilities enabled by the cloud architecture are utilized to provide different types of services: 
- Software as a Service (SaaS) is the service model where the capability provided to the consumer is the ability to use the cloud provider's applications running on a cloud infrastructure. The applications are accessible from various client devices through a thin client interface such as a web browser. Word processing and email applications such as Google Docs and Gmail or Customer Relationship Management (CRM) applications of salesforce.com are examples of this service model as well as backup, recovery and to some extent content delivery and publishing services.

- Platform as a Service (PaaS) is the service model where the capability provided to the consumer is a development or runtime environment, programming languages and application programming interfaces (APIs) to design and deploy infrastructure consumer created applications onto the cloud. Examples of these scalable services include Google App Engine, Microsoft Azure or force.com application development platforms.

- Infrastructure as a Service (IaaS) is the service model where the capability provided to the consumer is processing, storage, networks, and other fundamental computing resources. The consumer does not manage or control the underlying cloud hardware but has control over operating systems, storage, deployed applications, and possibly limited control of select networking components. These capabilities are delivered as a single server or as part of a collection of servers integrated into virtual machine environments. Amazon's Elastic Computing Cloud (EC2) is an example of IaaS.

\section{Impacts on Information Technology}

In order to discuss the economical impact of cloud computing, the following two economical market research studies can be given:

- In a recent report by Gartner forecasts worldwide cloud services market's revenue to surpass $\$ 68.3$ billion in 2010 and reach $\$ 148.8$ billion by 2014(Gartner Inc., 2010).

- $\quad$ Another recent study by IDC shows that Worldwide revenue from public IT cloud services exceeded $\$ 21.5$ billion in 2010 and will reach $\$ 72.9$ billion in 2015, representing a compound annual growth rate (CAGR) of $27.6 \%$ (IDC, 2012). This rapid growth rate is over four times the projected growth for the worldwide IT market as a whole $(6.7 \%)$.

- By 2015, one of every seven dollars spent on packaged software, server, and storage offerings will be through the public cloud model. The cloud movement is about much more than the cloud. Cloud cannot be sufficiently understood as a standalone phenomenon in the IT market, but rather as a core ingredient of a larger transformation of the IT industry - and many other industries using IT to transform them. Hence it is quite clear that although relatively new, cloud computing is already a very significant part of the IT industry (IDC, 2012).

In deciding whether hosting a service in the cloud makes sense over the long term, it was argued that the fine grained economic models enabled by Cloud Computing make tradeoff decisions more fluid, and in particular the elasticity offered by clouds serves to transfer risk (Amburst, et. al, 2009). Although hardware resource costs continue to decline, they do so at variable rates; for example, computing and storage costs are falling faster than WAN costs. Cloud computing can track these changes - and potentially passes them through to the customermore effectively than building one's own datacenter, resulting in a closer match of expenditure to actual resource usage.

\subsection{Adopting the Cloud}

Deciding to move an existing service to the cloud depends on

- the expected average and peak resource utilization, especially if the application may have highly variable spikes in resource demand;

- the practical limits on real-world utilization of purchased equipment; and

- various operational costs that vary depending on the type of cloud environment being considered.

Although the economic appeal of Cloud Computing is often described as "converting capital expenses to operating expenses" (CapEx to OpEx), we believe the phrase "pay as you go" more directly captures the economic benefit to the buyer. Hours purchased via Cloud Computing can be distributed non-uniformly in time (e.g., use 100 server-hours today and no server-hours tomorrow, and still pay only for what you use); in the networking community, this way of selling bandwidth is already known as usage-based pricing. In addition, the absence of up-front capital expense allows capital to be redirected to core business investment. An example to elaborate on the elasticity is given in (Amburst, et. al, 2009). 
As argued in (CloudU, 2011), OpEx is beneficial for the organization, as it gives it the flexibility to terminate costs at will. With a capital purchase, the server or software being acquired is fully committed to. Regardless of whether it is being utilized, the ongoing costs still need to be borne. Contrast this with OpEx where, in the event that the item is no longer required, payments can cease rapidly. It is for this reason that many companies prefer leasing vehicles in place of purchasing them outright. The findings are given in Table 1 for a sample comparison between an internal IT, managed services (similar to SaaS), and the cloud. 29\% has been gained with the use of the cloud for this typical organization.

\begin{tabular}{|c|c|c|c|}
\hline & Internal II & Managed Services & The Cloud \\
\hline Capital Investment & $\$ 40,000$ & $\$ 0$ & $\$ 0$ \\
\hline Setup Costs & $\$ 1,000$ & $\$ 5,000$ & $\$ 1,000$ \\
\hline Monthly Services & \$o & $\$ 4,000$ & $\$ 2,400$ \\
\hline Monthly Labor & $\$ 3,200$ & $\$ 0$ & $\$ 1,000$ \\
\hline Cost over three years & $\$ 149,000$ & $\$ 129,000$ & $\$ 106,000$ \\
\hline Savings Gained & $0 \%$ & $13 \%$ & $29 \%$ \\
\hline
\end{tabular}

Table 1. Estimated costs of infrastructure for two application servers, two database servers and a load balancer across internal, managed and Cloud deployment models. Source: (CloudU, 2011).

The primary cost of SaaS generally depends on the money spent to the data center. Hamilton presented a study to compare the costs of establishing and administering data centers (Hamilton, 2008). As shown in Table 2, for a medium-sized data center, the network usage requires 7.1 times more Money than that of very large data center. Similarly, the storage cost of medium-sized data center is 5.7 times higher than the storage cost of very large data center.

\begin{tabular}{|l|l|l|c|}
\hline Technology & Cost in Medium-sized DC & Cost in Very Large DC & Ratio \\
\hline Network & \$95 per Mbit/sec/month & \$13 per Mbit/sec/month & 7.1 \\
\hline Storage & \$2.20 per GByte / month & \$0.40 per GByte / month & 5.7 \\
\hline Administration & $\approx 140$ Servers / Administrator & $>1000$ Servers / Administrator & 7.1 \\
\hline
\end{tabular}

Table 2. Economies of scale in 2006 for medium-sized datacenter ( 1000 servers) vs. very large datacenter ( 50,000 servers) Sources: (Hamilton, 2008) and (Amburst, et. al, 2009).

An important gain of cloud computing in terms of data center costs is the fact that the risk of mis-estimating workload is shifted from the service operator to the cloud vendor. The cloud vendor may charge a premium (reflected as a higher use cost per server-hour compared to the 3-year purchase cost) for assuming this risk. Although there are sophisticated pricing models for infrastructure services (e.g., (Abramson, 2002), (Stuer, 2007)), the following simple equation is given in (Amburst, et. al, 2009):

$$
\text { UserHours }_{\text {cloud }} \times\left(\text { revenue }- \text { Cost }_{\text {cloud }}\right) \geq \text { UserHours }_{\text {datacenter }} \times\left(\text { revenue }-\frac{\text { Cost }_{\text {datacenter }}}{\text { Utilization }}\right)
$$

- The left-hand side multiplies the net revenue per user-hour (revenue realized per user-hour minus cost of paying Cloud Computing per user-hour) by the number of user-hours, giving the expected profit from using Cloud Computing.

- The right-hand side performs the same calculation for a fixed-capacity datacenter by factoring in the average utilization, including nonpeak workloads. Whichever side is greater represents the opportunity for higher profit.

- If Utilization $=1.0$ (the datacenter equipment is $100 \%$ utilized), the two sides of the equation look the same. In practice, the usable capacity of a datacenter (without compromising service) is typically 0.6 to 0.8 .

- The equation makes clear that the common element is the ability to control the cost per user hour of operating the service. 
Additionally, a cloud computing user can benefit from the resource usage on the scale of hours rather than years. First, unexpectedly scaling down (disposing of temporarily underutilized equipment) — for example, due to a business slowdown, or ironically due to improved software efficiency-normally carries a financial penalty. With 3-year depreciation, a \$2,100 server decommissioned after 1 year of operation represents a "penalty" of $\$ 1,400$. Cloud Computing eliminates this penalty (Amburst, et. al, 2009). For example, Intel Computing Services in 2000-2001 required negotiating a contract and longer-term use than per hour (Bayrak, 2011).

Moreover, technology trends suggest that over the useful lifetime of some purchased equipment, hardware costs will fall and new hardware and software technologies will become available. Cloud providers, who already enjoy economy-of-scale buying power, can potentially pass on some of these savings to their customers. If new technologies or pricing plans become available to a cloud vendor, existing applications and customers can potentially benefit from them immediately, without incurring a capital expense. In less than two years, Amazon Web Services increased the number of different types of compute servers ("instances") from one to five, and in less than one year they added seven new infrastructure services and two new operational support options(Amburst, et. al, 2009).

As a successful example, Elastic Compute Cloud (EC2) from Amazon Web Services (AWS) sells 1.0-GHz x86ISA "slices" for 10 cents per hour, and a new "slice", or instance, can be added in 2 to 5 minutes. Amazon's Scalable Storage Service (S3) charges $\$ 0.12$ to \$0.15 per gigabyte-month, with additional bandwidth charges of $\$ 0.10$ to $\$ 0.15$ per gigabyte to move data in to and out of AWS over the Internet. Amazon's bet is that by statistically multiplexing multiple instances onto a single physical box, that box can be simultaneously rented to many customers who will not in general interfere with each others' usage (Bayrak, 2011).

\subsection{New Business Models}

With the recent development to satisfy service-oriented computation to present the Internet as a utility to the end user, "tightly-coupled" business models have changed to so-called "loosely-coupled" business models. As an example to the tightly-coupled era, accepting credit card payments from strangers required a contractual arrangement with a payment processing service such as VeriSign or Authorize.net; the arrangement was part of a larger business relationship, making it onerous for an individual or a very small business to accept credit cards online. This was a typical business situation in earlier forms of the Internet.

However, in service-based era where SaaS has been perceived within the Internet, any individual can accept credit card payments with no contract, no long-term commitment, and only modest pay-as-you-go transaction fees, for example with the emergence of PayPal. The level of "touch" (customer support and relationship management) provided by these services are minimal to nonexistent, but the fact that the services are now within reach of individuals seems to make this less important.

Similarly, individuals' Web pages can now use Google AdSense to realize revenue from ads, rather than setting up a relationship with an ad placement company, which can be considered as a part of service-based business model. Moreover, individuals can distribute Web content using Amazon CloudFront rather than establishing a relationship with a content distribution network such as Akamai (Amburst, et. al, 2009).

Amazon Web Services capitalized on this insight in 2006 by providing pay-as-you-go computing with no contract: all customers need is a credit card. A second innovation was selling hardware-level virtual machines cycles, allowing customers to choose their own software stack without disrupting each other while sharing the same hardware and thereby lowering costs further (Amburst, et. al, 2009).

The development of an effective technology to make micropayments for content and services has the potential to revolutionize the web. In practice, however, consumers already do make micropayments indirectly by giving up personal information to providers of cloud services in exchange for access. For example, Facebook is an SaaS that provides storage and networking services to its users. Users have a choice of how much information to give to the site, such as one's email address, physical address, religion, age, relationship status, pictures of you and your friends, links to content you like, content you have created, etc.. The further a user reveals information, the more sharing he can do with his friends. Similarly, when we use Google, our searches are tracked and indexed, Hulu and Netflix create records of our viewing habits, Visa and MasterCard know how much liquor we buy, Ebay, Expedia, and especially Amazon know a great deal about our consumption habits, and many other sites deposit tracking cookies (Amburst, et. al, 2009). Hence, a new business model based on data analytics has been evolved, in which content and service providers gain more money by selling or renting the accumulated information on a user to third parties, or improving the services that they provide to the end user.

\subsection{Cost-based Evaluation}

The information technology revolution of the 80's and 90's in part replaced human workers with computers and automated systems. Hence, variable costs are replaced by fixed costs. This hardened the entry of the entrepreneurs having not much Money to the business. In opposition to that, cloud computing revolution has the opposite effect. Companies no longer need to build server farms and computing services become pay-as-you-go. This is especially significant in sectors where most costs are computing services such as content delivery, web based gaming and entertainment, social networking and so on. Since companies do not need to spend high 
amounts to legacy systems, they can build integrated cloud applications from the ground up. These factors make it easier for entrants to offer entertainment and other similar services. Since scale increases both revenue and costs at the same time, profitability can be maintained at variety of scales. This in turn allows new companies to offer services of particular interest to relatively small groups and also to scale up rapidly to take advantage of network externalities if they exist. Thus, the value of the intellectual property of large incumbents is reduced (Amburst, et. al, 2009).

Another economic issue of concern is that bankruptcy, reorganizations or even changes in business focus on the part of cloud service provides pose significant risks for users. In the worst case, users may lose data stored in the cloud. At a less catastrophic level, features and functionality may be dropped or no longer supported, service levels of companies distracted by internal problems may decline. This further weakens the company, and induces more customers to leave. The dynamics are very much like a bank-run (Amburst, et. al, 2009).

\subsection{State of Turkey}

In a recent research by Accenture, $8 \%$ of the small and medium scale companies keen on using cloud computing, this accumulates into more than 200 thousand and \$105 billion in the market (Küstür, 2013). The Money that these firms can invest for these services is $\$ 509$ per month on the average. As long as the trend of IT industry in Turkey continues, it was forecasted that by 2015, the market will have $\$ 139$ billion, as stated in the same research.

The "interested" sectors in Turkey are Retail-Trade and Transportation-Storage, and the ratios and cumulative market sizes are around $40 \%$ and $14 \%$, and $\$ 36$ billion and $\$ 10$ billion, respectively. It was stated in this research that the reason why only these sectors are interested is the recent advances in digital commerce. Moreover, the companies in these sectors consider reliability first, and than the cost, while choosing cloud provides. Some of these companies has already been started business with telecom operators and banking sector companies via cloud. This business model can be an example of the hybrid cloud as mentioned in Section 2.1.

As discussed further, the findings of the research in (Küstür, 2013) show that the highest percentage, 75\%, of the companies tend to adopt cloud computing in Turkey is due to the companies that are more than 9 years old or the ones that are newly established. What is important behind the reason why the companies refuse to use cloud computing lies in the fact that $40 \%$ have objections on the data privacy and security of cloud services? $36 \%$ of the companies refuse because they also refuse to change their acting model of business. It is clearly seen from the findings of the research that the use of cloud services will increase if the companies are trained such that new technology can be utilized to increase productivity, efficiency, and the company-owned data can be secured. An estimate based on these states that the potential market has $\$ 214$ billion by 2015 .

Other than this research, there is an ongoing work between Beykent University and Crede investigating the use of data analytics among companies. The work has been supported in part by TÜBITTAK, and based on the interviews that we have had up to this time, the companies in Turkey are really enthusiastic to the new technological trends, so as Cloud Computing.

\section{Conclusion}

In this study, it is intended to highlight the positive economical impacts of cloud computing for companies, which can be assessed by focusing on their business goals to make more profit. Although the definition of the term is unclear, the achievements of cloud computing are widely perceived by the industry both local and global. Under the cloud computing model, firms can rent as many virtual machines as they need at any given time, and either design or use off-the-shelf solutions to integrate company-wide data and then easily distribute access to users within and outside of the company. Hence, cloud computing converts fixed capital costs to variable costs, prevents under or over provisioning, and allows minute by minute flexibility. As a result of that, the companies concentrate on their own business goals on top on a stable and effective information technology backbone.

In this paper, we first discuss the basics of the cloud computing along with its implications on information technology (IT) field, with paying special attention to economical aspects. The impacts during the adoption of cloud computing, the chances to create new business models and cost-based analysis on the use of cloud computing services are given. Last, we put some basic findings on the status of Turkey. It is clear that, as the global companies do, the companies in Turkey are enthusiastic to the new technological trends. Although there are objection on the data privacy, it can be eliminated via technological training.

\section{References}

- Abramson, D., Buyya, R., and Giddy, J. 2002, “A computational economy for grid computing and its implementation in theNimrod-G resource broker.", Future Generation Computer Systems, 18, 8, 1061-1074.

- Armbrust, Michael, Fox, Armando, Griffith, Rean, Joseph, Anthony D., Katz, Randy H., Konwinski, Andrew, Lee, Gunho, Patterson, David A., Rabkin, Ariel, Stoica, Ion and Zaharia, Matei, 2009, "Above the 
Clouds: A Berkeley View of Cloud Computing”, UCB/EECS-2009-28,

http://www.eecs.berkeley.edu/Pubs/TechRpts/2009/EECS-2009-28.html.

- Bayrak, Ergin, Conley, John Patrick and Wilkie, Simon, 2011, "The Economics of Cloud Computing", No 1118, Vanderbilt Uni. Dept of Economics, http://EconPapers.repec.org/RePEc:van:wpaper:1118.

- Cheng, D. 2008, "PaaS-onomics: A CIO's Guide to using Platform-as-a-Service to Lower Costs of Application Initiatives While Improving the Business Value of IT". Technical Report, LongJump.

- CloudU, 2011, "Cloudonomics: the Economics of Cloud Computing", Rackspace Inc., http://www.techrepublic.com/resource-library/whitepapers/cloudonomics-the-economics-of-cloudcomputing/.

- Gartner, Inc., 2010, Press release: “Gartner Says Worldwide Cloud Services Market to Surpass $\$ 68$ Billion in 2010", http://www.gartner.com/it/page.jsp?id=1389313.

- Hamilton, J. 2008, “Internet-Scale Service Efficiency”, In Proc. Of Large-Scale Distributed Systems and Middleware (LADIS) Workshop, September.

- Hancock, Ian and Hutlay, Nikki, 2012, "Modelling the Economic Impact of Cloud Computing", KPMG Report, http://www.kpmg.com/au/en/issuesandinsights/articlespublications/pages/modelling-economicimpact-cloud-computing.aspx.

- IDC, 2012, “Cloud Research”, http://www.idc.com/prodserv/idc_cloud.jsp.

- Jackson, Kevin L., 2011, "The Economic Benefit of Cloud Computing", Forbes, http://www.forbes.com/sites/kevinjackson/2011/09/17/the-economic-benefit-of-cloud-computing/

- Küstür, S., 2013, “Accenture'a göre Türkiye bulut bilişism pazarı”, Teknobulut, http://bulut.teknoblog.com/2013/10/10/turkiye-bulut-bilisim-pazari-aylik-potansiyel.html.

- National Institute of Standards and Technology (NIST), 2014, "Definition of Cloud Computing", http://www.nist.gov/itl/cloud/index.cfm.

- Parkhill, D., 1966, The Challenge of the Computer Utility. Addison-Wesley Educational Publishers, US.

- Siegele, L. 2008, "Let It Rise: A Special Report on Corporate IT”, The Economist, October.

- Stuer, G., Van Mechelen, K., and Broeckhove, J. 2007,“A commodity market algorithm for pricing substitutable Gridresources”, Future Generation Computer Systems, 23, 5, 688-701. 